\title{
Some Aspects of Spinodal Decomposition in Spinel Solid Solutions
}

\author{
N. Burkert ${ }^{a}$, R. Grüne ${ }^{a}, H$. Schmalzried ${ }^{a}$, and S. Rahman ${ }^{b}$ \\ a Institut für Physikalische Chemie und Elektrochemie der Universität Hannover, Callinstraße 3A, \\ 30167 Hannover \\ b Institut für Mineralogie der Universität Hannover, 30167 Hannover
}

Z. Naturforsch. 50a, 525-532 (1995); received December 16, 1994

\author{
Dedicated to Professor Dr. E. Wicke on occasion of his 80 th birthday
}

\begin{abstract}
Decomposition morphologies of supersaturated spinel solid solutions in the quasi-binary system $\mathrm{Co}_{2} \mathrm{TiO}_{4}-\mathrm{CoAl}_{2} \mathrm{O}_{4}$ at $973 \mathrm{~K}$ show all pertinent features of spinodal decomposition. Since the decomposition morphology may not be considered as sufficient evidence for a spinodal process, the thermodynamics of the spinel solid solutions and the linear transport theory of spinodal decomposition were combined to corroborate the experimental results of the early decomposition reaction.

Simultaneous ordering, a common property of ternary and higher nonequilibrium solid solutions, was also observed. These ordering processes occur locally in distinct regions of the spinel crystal. Moreover, the spinodal wavelength $\lambda$ increases in time according to $\lambda \propto t$, which is unusual and will be discussed in the light of cation diffusion in semiconducting oxides with two cation sublattices.
\end{abstract}

\section{Introduction}

In a previous brief communication [1] we have presented preliminary results of an investigation on chemical reactions in supersaturated oxide solid solutions with spinel structure, the end members of which were $\mathrm{Co}_{2} \mathrm{TiO}_{4}$ and $\mathrm{CoAl}_{2} \mathrm{O}_{4}$, respectively. The spinel structure offers two sublattices to the cobalt, aluminium and titanium ions, so that a complex process of ordering and unmixing takes place in the course of equilibration. Experimentally it has been found that both reaction modes will occur. The unmixing takes place as spinodal demixing after the system has been undercooled into the region of the coherent spinodal [2]. In this paper we give the results and present the thermodynamic basis of their discussion in more depth.

\section{Spinodal Decomposition}

In his classic treatment Gibbs [3] formulated general conditions necessary for the stability of phases. Specifically, the boundary of the unstable region in a (quasi)-binary phase diagram is given analytically by setting the second derivative with respect to the mole fraction $x$ of the molar Gibbs energy $G$ (or the molar Gibbs energy of mixing $\Delta G$, respectively) equal to zero:

$$
\left(\frac{\partial^{2} G}{\partial x^{2}}\right)_{P, T}=\left(\frac{\partial^{2} \Delta G}{\partial x^{2}}\right)_{P, T}=0
$$

Reprint requests to Prof. Dr. H. Schmalzried.
Equation (1) represents the thermodynamic definition of the so-called "chemical spinodal" in solid solutions. If spinodal decomposition is considered in crystals, crystallographic constraints that lead to thermodynamic effects have to be taken into account. According to Cahn [4] one must incorporate the appropriate elastic free energy which modifies the stability condition:

$$
\left(\frac{\partial^{2} G}{\partial x^{2}}\right)_{P, T}+2 V_{\mathrm{m}} \eta^{2} Y_{[h k l]}=0,
$$

where $V_{\mathrm{m}}$ is the molar volume, $Y_{[h k l]}$ is the elastic modulus and $\eta$ describes the strain per unit concentration [5]. The additional strain energy contribution shifts the spinodal to lower temperatures which then is termed "coherent spinodal". Moreover, (2) shows that several coherent spinodals may exist, which depend on the crystallographic directions $[h k l]$ of the coherent intergrowth. Since no thermodynamic barrier exists for a system inside the coherent spinodal, the evolution of decomposition is determined by diffusion only.

The kinetic theory of spinodal decomposition starts from linear irreversible thermodynamics. The flux equation for the neutral particles of sort 2 in an isothermal, isobaric, isotropic and single-phase crystalline binary solid solution may be written in the form

$j_{2}=-\frac{D_{2} c_{2}}{R T} \nabla \mu_{2}=-\frac{D_{2} x_{2}\left(1-x_{2}\right)}{V_{m} R T} \nabla\left(\mu_{2}-\mu_{1}\right)$,

where the flux $j_{2}$ is referred to the lattice frame [6]. The driving force for interdiffusion is the gradient of the difference in chemical potentials $\mu_{i}$. Departures from

0932-0784 / 95 / 0600-0525 \$ 06.00 C) - Verlag der Zeitschrift für Naturforschung, D-72027 Tübingen 
Fick's law are due to steep gradients in composition and to the elastic strain energy which evolve during decomposition. Hilliard [5] has given an expression for the chemical potential which includes all the relevant contributions:

$$
\begin{aligned}
\mu_{2}-\mu_{1}= & \left(\frac{\partial G}{\partial x_{2}}\right)_{P, T}-2 \varkappa \nabla^{2} x_{2} \\
& +2 V_{\mathrm{m}} \eta^{2} Y_{[h k l]}\left(x_{2}-x_{2}^{0}\right) .
\end{aligned}
$$

The gradient energy coefficient $x$ follows from the free energy formalism of an inhomogeneous solid solution due to Cahn and Hilliard [7]. Its magnitude may be parameterized by defining an interaction distance $\Psi$ such that

$$
\varkappa=R T_{\mathrm{c}} \Psi^{2},
$$

where $T_{\mathrm{c}}$ is the critical temperature of unmixing [4]. A zeroth-order estimate correlates $\Psi$ with the average interatomic distance $r_{0}$ in the crystal structure [5]:

$$
\Psi=r_{0} / \sqrt{3} .
$$

One obtains the time-dependent diffusion equation by applying the continuity condition $\dot{c}_{2}=-\nabla j_{2}$ to (3) in combination with (4). If the inhomogeneities are not pronounced, the diffusion equation may be linearized, leading to

$$
\begin{aligned}
\dot{c}_{2}= & \frac{D_{2} x_{2}\left(1-x_{2}\right)}{V_{\mathrm{m}} R T} \\
& \cdot\left\{\left[\left(\frac{\partial^{2} G}{\partial x^{2}}\right)_{P, T}+2 V_{\mathrm{m}} \eta^{2} Y_{[h k l]}\right] \nabla^{2} x-2 x \nabla^{4} x\right\} .
\end{aligned}
$$

The time-dependence of the local change in composition $c_{2}$ is obtained from (7) as

$$
c_{2}(t)-c_{2}(t=0) \propto \exp (\mathscr{R}(\lambda) t) \cos \left(\frac{2 \pi}{\lambda} r\right),
$$

i.e. the concentration profile is given by a superposition of harmonic, plane waves of concentration with wavelength $\lambda$. The amplification factor $\mathscr{R}(\lambda)$ plays a central role in the linear theory of spinodal decomposition. It can be expressed as follows

$$
\begin{aligned}
\mathscr{R}(\lambda)= & -\frac{D_{2} x_{2}\left(1-x_{2}\right)}{R T} \\
& \cdot\left[\left(\frac{\partial^{2} G}{\partial x_{2}^{2}}\right)_{P, T}+2 V_{\mathrm{m}} \eta^{2} Y_{[h k l]}+2 \varkappa\left(\frac{2 \pi}{\lambda}\right)^{2}\right]\left(\frac{2 \pi}{\lambda}\right)^{2} .
\end{aligned}
$$

Because of the exponential dependence of the growth kinetics of composition waves on $\mathscr{R}(\lambda)$ the decomposition morphology will quickly be dominated by waves $\lambda_{\mathrm{m}}$ which correspond to the largest amplification factor:

$\lambda_{\mathrm{m}}=\left\{-\left[\left(\frac{\partial^{2} G}{\partial x_{2}^{2}}\right)_{P, T}+2 V_{\mathrm{m}} \eta^{2} Y_{[h k l]}\right] 16 \pi^{2} x\right\}^{-1 / 2}$

It can also be seen from (8) and (9) that essentially only the coherent spinodal with the smallest elastic modulus has to be taken into account in dealing with spinodal decomposition kinetics. In cubic crystals this normally is the [100]-spinodal. Therefore, $\mathscr{R}(\lambda)$ induces a twofold selection, namely with regard to the possible wavenumbers and the crystallographic directions.

\section{Thermodynamics of $\mathrm{Co}_{1+x} \mathrm{Al}_{2-2 x} \mathrm{Ti}_{x} \mathrm{O}_{4-}$ Spinel Solid Solutions}

The end members of the spinel solid solution which we consider here, i.e. $\mathrm{Co}_{2} \mathrm{TiO}_{4}$ and $\mathrm{CoAl}_{2} \mathrm{O}_{4}$, have different cation distributions. $\mathrm{Co}_{2} \mathrm{TiO}_{4}$ is a perfect inverse spinel $\left(\mathrm{Co}_{\mathrm{t}}^{2+} \mathrm{Co}_{\mathrm{o}}^{2+} \mathrm{Ti}_{\mathrm{o}}^{4+} \mathrm{O}_{4}\right)$, whereas the (ideal) normal cation distribution of $\mathrm{CoAl}_{2} \mathrm{O}_{4}$ is subject to a temperature-dependent inversion. Thus, the thermodynamics of the $\mathrm{Co}_{1+x} \mathrm{Al}_{2-2 x} \mathrm{Ti}_{x} \mathrm{O}_{4}$-solid solutions are mainly governed by the degree of inversion $\gamma$ according to the following equations:

$$
\begin{aligned}
(\mathrm{Co})_{\mathrm{t}}\left(\mathrm{Co}_{x} \mathrm{Al}_{2-2 x} \mathrm{Ti}_{x}\right)_{\mathrm{o}} \mathrm{O}_{4} & \\
& \rightleftharpoons\left(\mathrm{Co}_{1-\gamma} \mathrm{Al}_{\gamma}\right)_{\mathrm{t}}\left(\mathrm{Co}_{x+\gamma} \mathrm{Al}_{2-2 x-\gamma} \mathrm{Ti}_{x}\right)_{\mathrm{o}} \mathrm{O}_{4} \\
\mathrm{Co}_{\mathrm{t}}^{x}+\mathrm{Al}_{\mathrm{o}}^{x} & \rightleftharpoons \mathrm{Co}_{\mathrm{o}}^{\prime}+\mathrm{Al}_{\mathrm{t}}^{\prime} .
\end{aligned}
$$

The symbols agree with the notation of Kröger and Vink. The point defect reaction equation (12) describes the cation exchange in a phenomenological way. The corresponding microscopic processes may be quite complex. They depend on the intensive thermodynamic variables and the cation distribution itself.

As mentioned in a previous paper [1], the molar Gibbs energy change $\Delta \mathscr{G}(x, \gamma, T)$ reflecting the cation rearrangement (11) may be calculated if one uses the model of spinel solid solutions given by O'Neill and Navrotsky [8]:

$$
\begin{aligned}
\Delta \mathscr{G}(x, \gamma, T)= & \Delta \alpha \gamma+\beta \gamma^{2}-\Delta \zeta T \gamma \\
& +R T \sum_{i} b^{s} x_{i}^{s} \ln x_{i}^{s},
\end{aligned}
$$

where $\Delta \alpha, \beta$ and $\Delta \zeta$ are model parameters; $x_{i}^{s}$ is the fraction of cation $i$ on sublattice $s$ (given in Table 1), $b^{s}$ is the number of sites of type $s$ per formula unit. The 
Table 1. Site and material balances in the quasi-binary spinel solid solution $x \mathrm{Co}_{2} \mathrm{TiO}_{4}-(1-x) \mathrm{CoAl}_{2} \mathrm{O}_{4}$. The symbol $x_{i}^{s}$ denotes the fraction of cation $i$ on sublattice $s(s=\mathrm{t}, \mathrm{o})$, $\gamma$ is the degree of inversion; $t$ and o designate tetrahedral and octahedral, respectively.

\begin{tabular}{llll}
\hline$i$ & $x_{i}^{\mathrm{t}}$ & $2 x_{i}^{\mathrm{o}}$ & $\sum$ \\
\hline $\mathrm{Co}^{2+}$ & $1-\gamma$ & $x+\gamma$ & $1+x$ \\
$\mathrm{Al}^{3+}$ & $\gamma$ & $2-2 x-\gamma$ & $2-2 x$ \\
$\mathrm{Ti}^{4+}$ & 0 & $x$ & $x$ \\
$\Sigma$ & 1 & 2 & 3 \\
\hline
\end{tabular}

equilibrium condition $(\partial \Delta \mathscr{G}(x, \gamma, T) / \partial \gamma)_{x, T}=0$ allows to calculate $\gamma(x, T)$ from

$$
R T \ln \frac{\gamma(x+\gamma)}{(2-2 x-y)(1-\gamma)}+\Delta \alpha+2 \beta \gamma-\Delta \zeta T=0 .
$$

Equation (14) is solved numerically for $0 \leq x \leq 1$ and temperatures between $973 \mathrm{~K}$ and $1773 \mathrm{~K}$. Consequently, the molar free Gibbs energy of mixing $\Delta G(x, T)$ is known.

$$
\begin{aligned}
\Delta G(x, T)= & \Delta \mathscr{G}(x, T)-x \Delta \mathscr{G}(1, T) \\
& -(1-x) \Delta \mathscr{G}(0, T)+W x(1-x) .
\end{aligned}
$$

Because normally the endmembers of a solid solution have different molar volumes, an additional elastic energy term has been introduced into (15). Using the elastic interaction parameter $W$ this term is approximated in analogy to a regular solution model. All model parameters can be obtained from the fitting procedure. For different sets of parameters, the Gibbs energies have been calculated and the corresponding theoretical miscibility gaps have been deduced by using the common tangent construction. The miscibility gap which is in best agreement with experimental results is shown in Figure 1. The model parameters chosen $\left(\Delta \alpha=63 \mathrm{~kJ} \mathrm{~mol}^{-1}, \beta=-20 \mathrm{~kJ} \mathrm{~mol}^{-1}, W=55 \mathrm{~kJ}\right.$ $\mathrm{mol}^{-1}$ ) differ slightly from those of earlier calculations [1] and correspond to theoretical predictions [9].

\section{Results}

\subsection{Theoretical Results}

The theoretical concepts outlined in the preceeding sections allow us to calculate the chemical and coherent [100]-spinodal in the quasi-binary system $\mathrm{Co}_{2} \mathrm{TiO}_{4}-\mathrm{CoAl}_{2} \mathrm{O}_{4}$. They are illustrated graphically in Figure 1. The spinodal curves were determined analytically from (1) and (2) using the elastic constants in

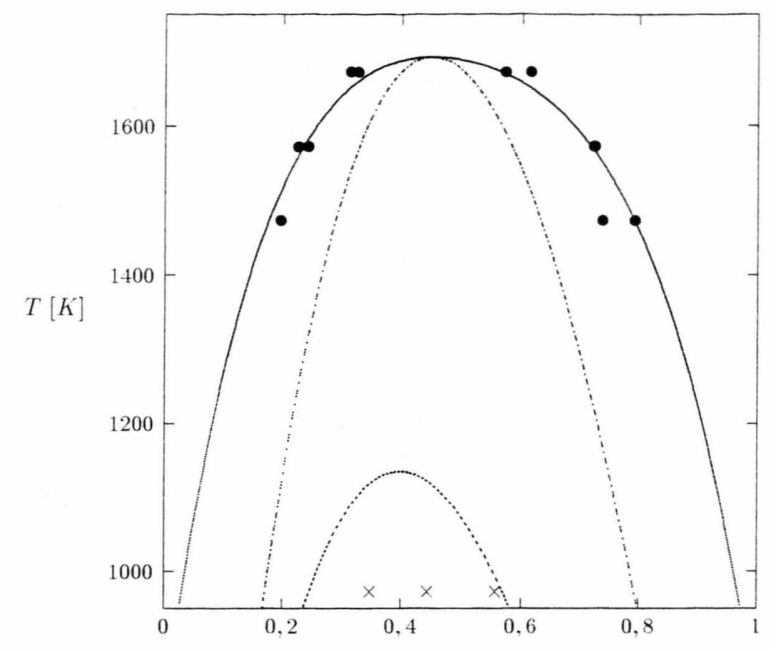

Fig. 1. Calculated phase diagram of the quasi-binary system $\mathrm{Co}_{2} \mathrm{TiO}_{4}-\mathrm{CoAl}_{2} \mathrm{O}_{4}$. Dash-dot line = calculated chemical spinodal. Dashed line = calculated coherent [100]-spinodal. Dots represent experimental data from phase equilibria determination. Crosses $(x)$ represent homogenized spinel solid solutions, decomposed isothermally at $973 \mathrm{~K}$ and studied at room temperature.

Table 2. Elastic constants $C_{i i}$ and temperature derivatives $\tau_{i i}$ of the elastic constants which have been used to calculate the coherent spinodal in the $\mathrm{Co}_{2} \mathrm{TiO}_{4}-\mathrm{CoAl}_{2} \mathrm{O}_{4}$-solid solution according to (2).

\begin{tabular}{llc}
\hline & $\mathrm{CoAl}_{2} \mathrm{O}_{4}$ & $\mathrm{Co}_{2} \mathrm{TiO}_{4}$ \\
\hline$C_{11}[\mathrm{GPa}]$ & 290.5 & 267.6 \\
$C_{12}[\mathrm{GPa}]$ & 170.3 & 105.6 \\
$C_{44}[\mathrm{GPa}]$ & 138.6 & 95.3 \\
\hline
\end{tabular}

[12] Values of $\mathrm{Fe}_{3} \mathrm{O}_{4}[12]$

\begin{tabular}{ll}
\hline$\tau_{11}\left[\mathrm{GPa} \mathrm{K}^{-1}\right]$ & $-262 \times 10^{-4}$ \\
$\tau_{12}\left[\mathrm{GPa} \mathrm{K}^{-1}\right]$ & $-997 \times 10^{-5}$ \\
$\tau_{44}\left[\mathrm{GPa} \mathrm{K}^{-1}\right]$ & $-878 \times 10^{-5}$ \\
\hline
\end{tabular}

Values of $\mathrm{MgAl}_{2} \mathrm{O}_{4}$ [13]

Table 2, which have been averaged according to Vegard's law [10]. In contrast to our earlier calculations, this time the temperature dependence of the elastic constants and their concomitant effect on the anisotropy has been included [11]. However, the temperature dependence of the lattice parameter $a$ is neglected, therefore $\eta=(\partial \ln a / \partial x)_{P, T}$ is obtained directly from lattice parameter measurements (Figure 2). The second derivative of the Gibbs energy of mixing $\Delta G$ has been calculated analytically from (15). 


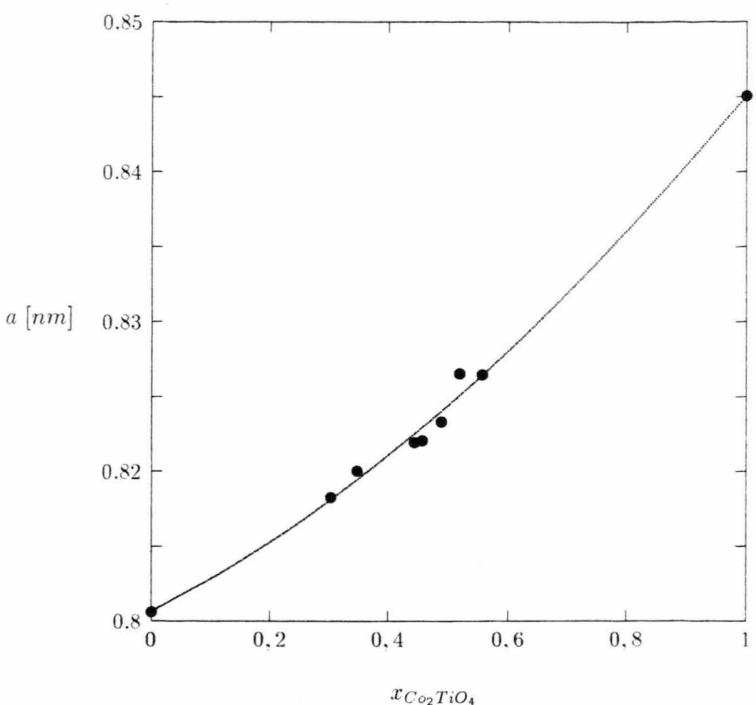

Fig. 2. Room temperature lattice constants for $\mathrm{Co}_{2} \mathrm{TiO}_{4}-$ $\mathrm{CoAl}_{2} \mathrm{O}_{4}$-spinel solid solutions. Experimental data are represented by dots. The curve is obtained form a 3.-polynominal regression analysis.

The spinodal wavelength $\lambda_{\mathrm{m}}$ has been calculated as a function of temperature and composition. Three wavelengths as obtained from (10) are given in Table 3 along with the corresponding parameters at $T=973 \mathrm{~K}$. The gradient energy coefficient $x$ was obtained from (5) and (6); the interatomic distance $r_{0}$ was estimated by averaging linearly over the nearestneighbour cation distances in the spinel structure [14].
Table 3. Calculated spinodal wavelength $\lambda_{\mathrm{m}}$ at $T=973 \mathrm{~K}$ for $\mathrm{Co}_{2} \mathrm{TiO}_{4}-\mathrm{CoAl}_{2} \mathrm{O}_{4}$-spinel solid solutions. Additionally, the table shows all temperature- and composition-dependent crystallographic and elastic parameters which have been used.

\begin{tabular}{llll}
\hline$x_{\mathrm{Co}_{2} \mathrm{TiO}_{4}}$ & 0.35 & 0.45 & 0.55 \\
\hline$a[\mathrm{~nm}]$ & 0.8195 & 0.8227 & 0.8262 \\
$\eta$ & $3.728 \times 10^{-2}$ & $4.060 \times 10^{-2}$ & $4.377 \times 10^{-2}$ \\
$V_{\mathrm{m}}\left[\mathrm{m}^{3} \mathrm{~mol}^{-1}\right]$ & $4.14 \times 10^{-5}$ & $4.20 \times 10^{-5}$ & $4.25 \times 10^{-5}$ \\
\hline$\chi\left[\mathrm{J} \mathrm{m}^{2} \mathrm{~mol}^{-1}\right]$ & $5.06 \times 10^{-16}$ & $5.10 \times 10^{-16}$ & $5.16 \times 10^{-16}$ \\
\hline$\left(\partial^{2} \Delta_{\mathrm{mix}} G / \partial x^{2}\right)_{P, T}$ & -39185 & -44770 & -44703 \\
{$\left[\mathrm{~J} \mathrm{~mol}{ }^{-1}\right]$} & & & \\
\hline$Y_{[100]}[\mathrm{GPa}]$ & 255.73 & 259.23 & 262.31 \\
\hline$\lambda_{\mathrm{m}}[\mathrm{nm}]$ & 2.87 & 3.01 & 6.32 \\
\hline
\end{tabular}

\subsection{Experimental Results}

The details of the experiments have been explained in [1]. In what follows we present the pertinent results.

Figure 2 shows the composition dependence of the lattice parameter $a$ of $\mathrm{Co}_{2} \mathrm{TiO}_{4}-\mathrm{CoAl}_{2} \mathrm{O}_{4}$-spinel solid solutions. It shows higher negative deviations from ideality than that of the analogous system $\mathrm{Mg}_{2} \mathrm{TiO}_{4}$ $\mathrm{MgAl}_{2} \mathrm{O}_{4}$ [15]. At moderate temperatures it is known that pure $\mathrm{Mg}_{2} \mathrm{TiO}_{4}$ exhibits a large degree of shortrange order in the octahedral sublattice [16]. Replacing $\mathrm{Mg}^{2+}$ by the transition metal cation $\mathrm{Co}^{2+}$ we may assume that similar short-range ordering appears on a larger scale, which decreases the lattice constant.

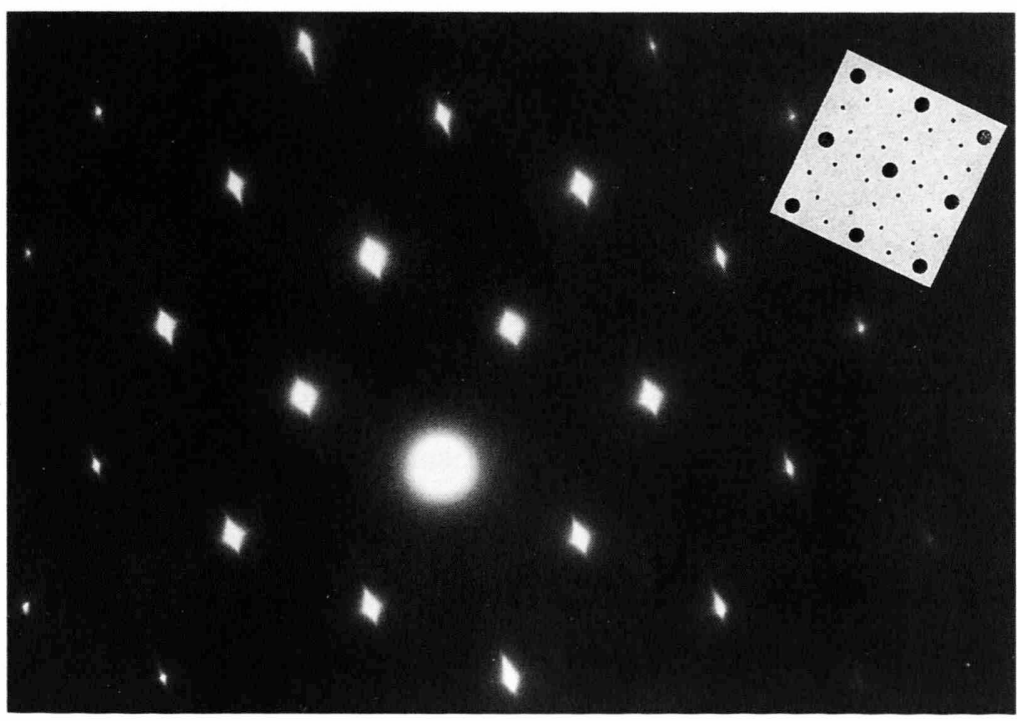

Fig. 3. Electron diffraction pattern (zone axis [100]) of a quenched sample (44.4 $\mathrm{mol}_{\%} \% 5.6 \mathrm{~mol}^{\%} \mathrm{CoAl}_{2} \mathrm{O}_{4}$ ) annealed at $973 \mathrm{~K}$ for $2 \mathrm{~d}$ showing spot streaking along [010] and [001], and superlattice reflections. 

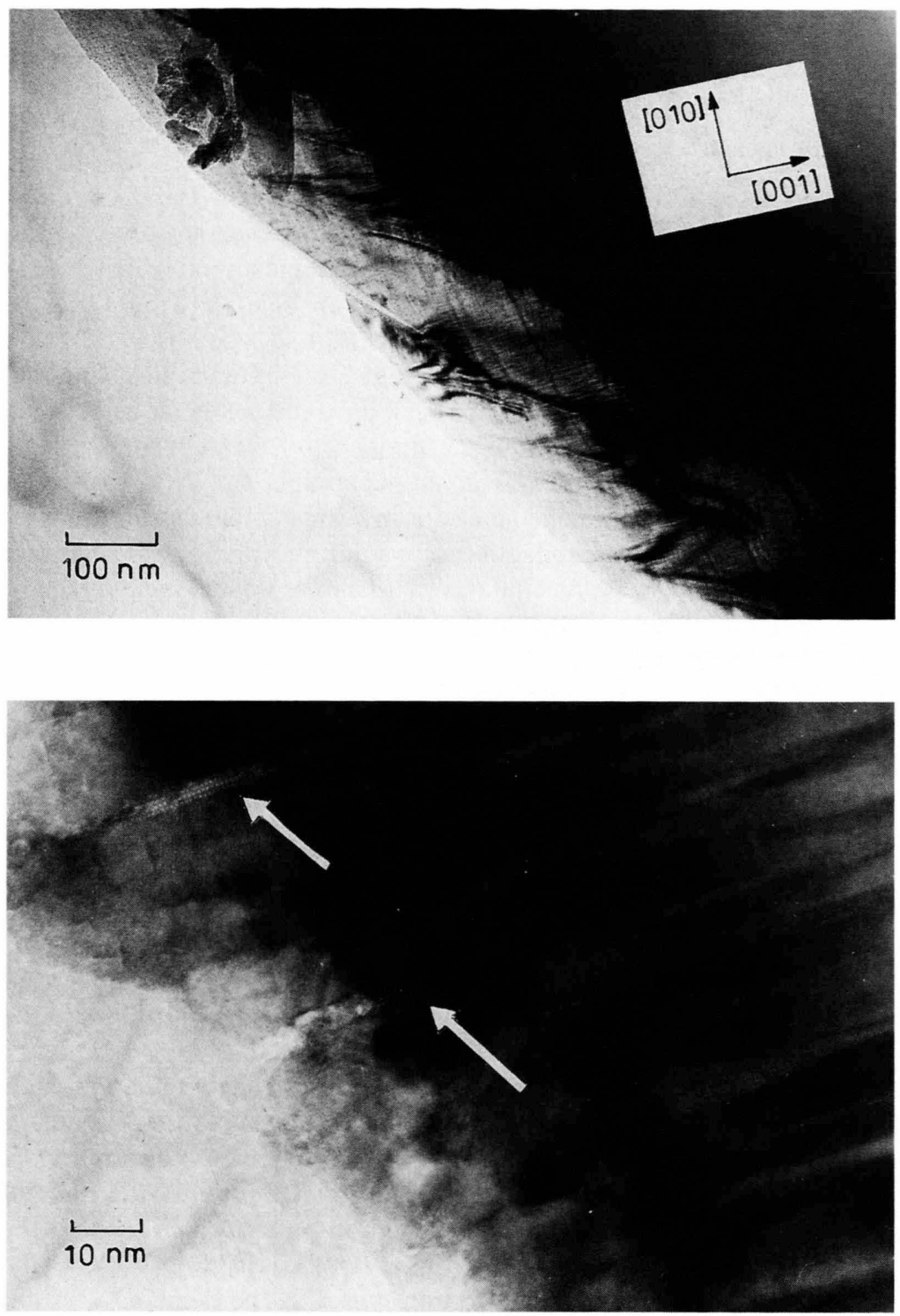

Fig. 4. Transmission electron micrograph which corresponds to the SADpattern of Figure 3. The periodic distribution of coherent platelets with ca. $5.5 \mathrm{~nm}$ thickness (aligned along [010]) can be seen.
Fig. 5. High resolution transmission electron micrograph of the specimen of Figs. 3 and 4. The demixed crystal shows locally ordered regions (arrowed). The lattice constant of the superstructure corresponds to the value of the homogeneous spinel solid solution.
Figures 3, 4, and 5 show the SAD-patterns and TEM-images of a sample $\left(45 \mathrm{~mol} \% \mathrm{Co}_{2} \mathrm{TiO}_{4} /\right.$ $55 \mathrm{~mol}_{0} \mathrm{CoAl}_{2} \mathrm{O}_{4}$ ) which has been annealed at $973 \mathrm{~K}$ for $2 \mathrm{~d}$, followed by quenching. They are comparable to the transmission electron micrograph given in [1]. The diffraction pattern (zone axis [001]) exhibits spot streaking along [100] and [010] due to the modulated structure shown in Figure 4. The microstructure after spinodal decomposition is characterized by a periodic distribution of coherent platelets of about $5.5 \mathrm{~nm}$ thickness, aligned along the elastically soft
[100] and [010]-directions. Moreover, diffuse superlattice reflections appear on the diffraction pattern. A high-resolution transmission electron micrograph of ordered regions of the demixed crystal is given in Figure 5. The lattice constant of the superstructure is identical to that of the homogeneous spinel solid solution.

All experimental data which concern the wavelengths of periodic concentration fluctuations are summarized in Table 4. Values are taken directly from the electron micrographs and/or are calculated from 
Table 4. Mean wavelengths $\lambda$ of composition periodicity, taken from electron diffraction patterns (SAD) and electron micrographs (TEM) in $\mathrm{Co}_{2} \mathrm{TiO}_{4}-\mathrm{CoAl}_{2} \mathrm{O}_{4}$-spinel solid solutions. Annealing temperature is $T=973 \mathrm{~K}$. The absolute error in $\lambda$ is $\Delta \lambda= \pm 1 \mathrm{~nm}$. Values marked by asterisk $\left({ }^{*}\right)$ are obtained from a linear extrapolation of the experimental data (see Fig. 4 in [1]).

\begin{tabular}{|c|c|c|c|c|c|c|}
\hline \multirow{3}{*}{$\begin{array}{l}x_{\mathrm{Co}_{2} \mathrm{TiO}_{4}} \\
\text { time [d] }\end{array}$} & \multicolumn{6}{|c|}{$\lambda[\mathrm{nm}]$} \\
\hline & \multicolumn{2}{|c|}{$34.8 \mathrm{~mol} \%$} & \multicolumn{2}{|c|}{$44.4 \mathrm{~mol} \%$} & \multicolumn{2}{|c|}{$55.7 \mathrm{~mol} \%$} \\
\hline & SAD & TEM & SAD & TEM & SAD & TEM \\
\hline 0 & \multicolumn{2}{|c|}{$1 *$} & 3 & 2 & \multicolumn{2}{|c|}{$6^{*}$} \\
\hline 1 & 3 & 2 & 4 & 4 & 5 & 6 \\
\hline 2 & & & 5 & 6 & & \\
\hline 4 & & & & 9 & & 7 \\
\hline 5 & 5 & 6 & & & & \\
\hline 8 & & 9 & & 16 & & 8 \\
\hline 15 & & 15 & & & & \\
\hline 24 & & & & & & 12 \\
\hline
\end{tabular}

the corresponding SAD-patterns [17]. If we write the hypothetical growth-law of the spinodal wavelength $\lambda$ in the form

$$
\lambda(t)=c \cdot\left(t-t_{0}\right)^{1 / m}+\lambda_{0},
$$

where $\lambda_{0}$ denotes the wavelength at $t_{0} \cong 0$, regression analysis on a double-logarithmic scale yields $m \approx 1$. Therefore, the spinodal wavelength varies approximately linearly within the chosen equilibration time. The homogenized and subsequently quenched spinel solid solutions of composition $34.8 \mathrm{~mol} \%$ and $55.7 \mathrm{~mol} \% \quad \mathrm{Co}_{2} \mathrm{TiO}_{4}$ have not been studied with TEM, nevertheless the spinodal wavelengths at $t_{0}=0$ could be obtained from a regression analysis. These values are also given in Table 4.

\section{Discussion}

As pointed out by de Fontaine [18], one criterion for spinodal decomposition in a (quasi)-binary solid solution follows from the thermodynamic definition as given in (2). Any diffusion-controlled phase transformation which occurs during the initial anneal inside the coherent spinodal must be spinodal decomposition. However, two points are worth mentioning in this context: 1 . The true coherent spinodal of a given system is normally unknown owing to the lack of pertinent thermodynamic data. 2. The thermodynamic concept itself can be questioned if the transition from nucleation to spinodal decomposition is gradual
$[2,19]$. Another criterion is a kinetic one: The continually growing composition waves as given by (8) are characteristic of spinodal decomposition. However, it is difficult to measure concentration fluctuations quantitatively on such a fine scale, i.e. below $\leq 10 \mathrm{~nm}$. From an experimental point of view the decomposition morphologies of the demixed crystal are taken as evidence for spinodal decomposition, which means that periodic precipitates are solely attributed to the spinodal demixing process. Also, one has to be aware that in systems with high strain $\eta$ the typical spinodal morphology will break down before it can be detected [20].

The microstructures of all three $\mathrm{Co}_{2} \mathrm{TiO}_{4}-\mathrm{CoAl}_{2} \mathrm{O}_{4}$ spinel solid solutions investigated here show the typical spinodal decomposition morphology. The size of spinel precipitates $(\approx 10 \mathrm{~nm})$ as well as their crystallographic orientation ([100]) agrees with theoretical considerations [2]. We can estimate the maximum average wavelength $\lambda^{*}$ of a coherent periodic composition modulation in a (quasi-)binary solid solution by following de Fontaine [20]. Taking into account the experimental data of the solid solution of critical composition $\left(\approx 45 \mathrm{~mol} \% \mathrm{Co}_{2} \mathrm{TiO}_{4}\right)$, the calculation gives $\lambda^{*} \approx 20 \mathrm{~nm}$. The experimental spinodal wavelengths seem to be lower than $\lambda^{*}$. Since the SAD-diffraction patterns exclusively show the reflexes of one of the equilibrated spinel solid solutions, we conclude that the decomposition proceedes coherently within the time-interval studied (Table 4).

Morral and Cahn [21] have shown that an outstanding feature of ternary one-sublattice systems is the possibility of the simultaneous occurance of spinodal decomposition and continuous ordering. Actually, the SAD-diffraction pattern in Fig. 3 and the corresponding HRTEM-image in Fig. 5 show the existence of superstructures in the demixed crystals. However, the same sample does not exhibit ordered precipitates. We conclude that the ordering processes take place locally in other regions of the crystal (Fig. $3 \mathrm{a} / 3 \mathrm{~b}$ in [1]). Rogalla and Schmalzried observed already that the re-equilibration of the cation distribution in $\mathrm{NiAl}_{2} \mathrm{O}_{4}$ cannot be described by simple exchange processes between the two sublattices of the spinel. They also found that the rate of cation redistribution is different in different regions of the crystal [22]. In the $\mathrm{Co}_{2} \mathrm{TiO}_{4}-\mathrm{CoAl}_{2} \mathrm{O}_{4}$-spinel solid solutions the tetrahedral and the octahedral sublattice are occupied by three sorts of cations. Then, in contrast to ternary metallic systems and simple spinels, multiple 
mechanisms have to be considered in order to describe the sublattice exchange processes. Because they are expected to occur simultaneously during spinodal decomposition, they may influence locally the demixing kinetics which leads to cation ordering. They may also be responsible for the unusual growth-rate of the spinodal wavelength as discussed below.

We mentioned in the beginning that the criteria for spinodal decomposition based on precipitate morphology are unreliable. Thus one has to consider theoretical concepts in addition. Because of the exponential dependence of the growth (or decay) kinetics of the composition waves (see (8)), the spinodal wavelength $\lambda_{0}$ may be approximated by $\lambda_{\mathrm{m}}$. The experimental values given in Tab. 4 indicate that $\lambda_{0}$ increases with increasing percentage of $\mathrm{Co}_{2} \mathrm{TiO}_{4}$. The theoretical prediction agrees with this result (see Table 3 ). Moreover, the calculated wavelengths $\lambda_{\mathrm{m}}$ agree reasonable with the experimental data. However, it should be noted that the only well known parameter given in Table 3 is the composition-dependent lattice constant of the spinel solid solution (Figure 1). Furthermore, the resulting strain $\eta$ dominates the calculation of $\lambda_{\mathrm{m}}$ owing to the quadratic term in (10). Therefore, the calculation based on the linear theory can explain the experimental results of the early decomposition kinetics quantitatively. From the calculated phase diagram we conclude that the homogeneous spinel solid solutions were decomposed within the phase field of the coherent spinodal (Figure 2). From a theoretical point of view these aspects may also be regarded as a criterion for spinodal decomposition.

In Table 4 and Fig. 4 of [1] it is shown that the growth rate of the spinodal wavelength depends on composition and is highest for a sample of the critical composition. Similar results were obtained for the metallic system $\mathrm{Cu}-\mathrm{Ni}-\mathrm{Fe}[23,24]$. It was presumed (see (3)) that the driving force for interdiffusion, i.e. the second derivative of the Gibbs energy of mixing $\Delta G$, is responsible for the different growth rates, although the elastic energy contribution and the gradient energy have to be taken into account. Cahn has already given an upper limit for the rate of decomposition at a fixed spinodal wavelength $\lambda_{\mathrm{m}}$ [25]. So far there exists no (microscopic) theory which can describe the experimental increase of the spinodal wavelength $\lambda$ in time [2]. Nevertheless, in solid mixtures there is ample experimental evidence for the validity of the WagnerLifshitz-Slyozov rate law $\lambda \propto t^{1 / 3}$. It is not clear yet whether the growth of the spinodal wavelength is due to a condensation-evaporation type of mechanism or whether it occurs by local doubling as suggested by de Fontaine [26]. Therefore, we restrict the discussion of the time-dependence of spinodal wavelengths to some qualitative considerations on decomposition in spinel solid solutions.

The experimental spinodal wavelength in the system $\mathrm{Co}_{2} \mathrm{TiO}_{4}-\mathrm{CoAl}_{2} \mathrm{O}_{4}$ varies linearly with time, which differs from other experimental data on spinodal decomposition. It is remarkable that a constant growth rate $(\dot{\lambda})$ is only found in fluids at the late stage of exsolution [27].

As has already been noticed in [1], the kinetics of spinodal decomposition in oxide solid solutions will be influenced by some characteristic features of diffusion in ionic crystals. In contrast to the traditionally studied metallic systems, ionic crystals are built from at least two sublattices. One sublattice is occupied by anions and may be considered here as a rigid frame, within which the smaller cations are dissolved and the coherent demixing processes will take place. We believe that the existence of two cation sublattices in the spinel structure is the main problem in the spinodal decomposition of the system $\mathrm{Co}_{2} \mathrm{TiO}_{4}-\mathrm{CoAl}_{2} \mathrm{O}_{4}$. Strictly speaking, any flux equation (as (3)) has to be formulated with structure elements which are the real diffusing species in the crystal lattice. Two distinct transport equations will result which describe the diffusional processes in each cation sublattice. The question is in which way the coupling of these fluxes of structure elements and their driving forces will occur [28]. A detailed microscopic formulation of the diffusion models leads to a complex system of non-linear differential equations. The solutions have to be analyzed carefully in order to decide which process governs the spinodal decomposition.

We further note that for ionic crystals the chemical potentials $\mu_{i}$ of (3) must be replaced by the electrochemical potentials $\eta_{i}$. Nernst-Planck type equations are the result of the coupling of the cation fluxes due to the electroneutrality condition. In semiconducting oxides, moreover, the coupling is due to the conservation of lattice sites. It can be shown that internal oxygen potential gradients build up during chemical diffusion [29]. Both modifications should have no effect on the result expressed by (9) [30]. However, if transition metal cations are involved, the oxygen potential gradient may disturb the redox equilibria, which will influence the cation arrangement between the spinel sublattices and thus the transport processes. And fi- 
nally, we repeat that we considered spinodal decomposition of the spinel solution only in the linear regime. Any ripening process complicates the formal treatment of exsolution, owing to the appearance of coherent phase boundaries.

Our discussion showed that equilibration processes in spinel solid solutions depend on a large number of system parameters. Therefore, comprehensive predictions about the thermodynamics and kinetics of ternary (or higher) systems with several sublattices are hardly possible yet. Experimental difficulties in the study of spinodal decomposition are essentially due to

[1] N. Burkert et al, Ber. Bunsenges. Phys. Chem. 96, 1602 (1992).

[2] K. Binder, Materials Science and Technology, J.W. Cahn, P. W. Haasen, and E. J. Kramer, eds., Vol. 5, VCH, Weinheim 1991.

[3] J.W. Gibbs, Collected Works, Vol. I, p. 105-115, 252258, Longmans, Green and Co., New York 1928.

[4] J.W. Cahn, Trans. AIME 242, 166, (1968).

[5] J. E. Hilliard, Phase Transitions, p. 497, Metals Park, Ohio 1968.

[6] H. Schmalzried, Solid State Reactions, 2. ed., Monographs in Modern Chemistry, Vol. 12, VCH, Weinheim 1981.

[7] J.W. Cahn and J. E. Hilliard, J. Chem. Phys. 28, 258 (1958).

[8] H. St. C. O'Neill and A. Navrotsky, Am. Mineral. 69, 733 (1984)

[9] H. E. Cook and D. de Fontaine, Acta Met. 19, 607 (1971).

[10] J. Shanker, R. Singh, and M. Kumar, Phys. Stat. Sol. B 156, 131 (1989).

[11] C. M. F. Jantzen and H. Herman, Phase Diagrams, A. M. Alper, ed., Vol. 5, Academic Press, New York 1978.

[12] Z. Li et al, J. Mater. Sci. 26, 2621 (1991).

[13] Z. P. Chang and G. R. Barsch, J. Geophys. Res. 78, 2418 (1973).

[14] R. J. Hill, J. R. Craig, and G. V. Gibbs, Phys. Chem. Miner. 4, 317 (1979). the fact that the finite time which is necessary for the quenching into the coherent spinodal phase field forbids the observation of the early stages of equilibration.

\section{Acknowledgement}

We are indebted to the Deutsche Forschungsgemeinschaft whose financial support is gratefully acknowledged. The Fonds der Chemischen Industrie gave valuable financial assistance.

[15] A. Muan, J. Hauck, and T. Löfall, Proc. Third Lunar Sci. Conf., Geochim. Cosmochim. Acta Suppl. 3, Vol. 1, 185 (1972).

[16] B. A. Wechsler and A. Navrotsky, J. Solid State Chem. 55, 165 (1984).

[17] G. Thomas, Diffraction and Imaging Techniques in Materials Science, S. Amelinckx, R. Gevers, and J. Van Landuyt, eds., Vol. 1, North-Holland, Amsterdam 1978.

[18] D. de Fontaine, Ultrafine Grain Metals, Proc. Sagamore Army Mater. Res. Conf. 16th, p. 93, 1970.

[19] H. Reiss, Ber. Bunsenges. Phys. Chem. 79, 943 (1975).

[20] D. de Fontaine, Acta Met. 17, 477 (1969).

[21] J. E. Morral and J. W. Cahn, Acta Met. 19, 1037 (1971).

[22] W. Rogalla and H. Schmalzried, Ber. Bunsenges. Physik. Chem. 72, 12 (1968).

[23] R. J. Livak and G. Thomas, Acta Met. 19, 497 (1971).

[24] V. M. Lopez et al, Acta Metall. Mater. 41, 265 (1993).

[25] J. W. Cahn, Acta Met. 9, 795 (1961).

[26] D. de Fontaine, Treatise of Solid State Chemistry, N. B. Hannay, ed., Vol. 5, Plenum Press, New York 1975.

[27] E. D. Siggia, Phys. Rev. A 20, 595 (1979).

[28] M. Martin, T. Pfeiffer and H. Schmalzried, Report, Institut für Physikalische Chemie und Elektrochemie, Universität Hannover 1988.

[29] H. Schmalzried and Th. Pfeiffer, Z. Physik. Chem. NF 148, 21 (1986).

[30] N. Burkert, Spinodale Entmischung in Mischphasen ternärer Oxide, Ph.D. thesis, Universität Hannover, 1993. 